\title{
Zur Arbeit kirchlicher AV-Medienzentralen
}

\author{
von Klaus Hinkelmann
}

Die Pastoralinstruktion Communio et Progressio über die Instrumente der sozialen Kommunikation intendierte weitgehende Veränderungen im Verhältnis der Kirche zu den Medien überhaupt. Sie brachte auch in der Bundesrepublik eine Neuordnung der Medienarbeit mit sich, unter anderem wurden nach 1970 verstärkt AV-Medienzentralen in den Diözesen eingerichtet, soweit nicht die alten Diözesanfilmstellen diese Aufgaben übernahmen.

Die inzwischen beachtlichen Ausleihzahlen dieser Einrichtungen ließen die kirchlichen AV-Medienzentralen zu einem Faktor werden, dem neben den anderen bewährten Distributionseinrichtungen für Medien ein eigener Stellenwert zukommt. Eine in letzter Zeit angefachte Diskussion um das Zueinander insbesondere von staatlichen AV-Medienzentralen ${ }^{1}$ kann dazu verhelfen, die Notwendigkeit eines Engagements in der Medienarbeit mit möglichst differenzierten Distributionsformen stärker publik zu machen.

\section{Grundlagen der Arbeit der Verleiborganisationen}

Geschäftsordnungen, Aufgabenbeschreibungen und schließlich Entleihvorschriften der Bildstellen benennen regelmäßig an erster Stelle die Schulen als Ziel- und Orientierungsgrößen².

Prinzipiell besteht eine gesetzliche Verpflichtung der Kommunen, die Schulen mit Lehrmitteln auszustatten; definitorisch werden auch die Medien dazugerechnet. Andererseits ist die Einrichtung von Kreis- und Stadtbildstellen den kommunalen Gebietskörperschaften als freiwillige Leistung anheimgestellt, so daß mit einer sehr unterschiedlichen Ausstattung der Bildstellen zu rechnen ist.

Die Ausrichtung der Bildstellen auf die Versorgung von Schulen aller Art ist also angelegt; die (organisierte) Jugend- und Erwachsenenbildung gemäß den Erwachsenenbildungsstellen spielt demgegenüber eine untergeordnete Rolle.

Die Zuständigkeit der Bildstellen auch für den konfessionellen Religionsunterricht ist $\mathrm{zu}$ Recht mit dem Verweis auf dessen Status als ordentliches Lehrfach ${ }^{3}$ an den Anfang der Überlegungen zu stellen. In der Tat ist von den Grundrechten und Grundwerten auszugehen, wie sie im Grundgesetz der Bundesrepublik Deutschland und in den Länderverfassungen festgeschrieben sind. Zu nennen sind hier insbesondere Grundgesetz Artikel 3, der „die Freiheit des Glaubens, des Gewissens und die Freiheit des religiösen und weltanschaulichen Bekenntnisses " ${ }^{\text {" }}$ garantiert. Gemäß Grundgesetz Artikel 4 hat die Religionsausübung ungestört zu bleiben; Artikel 7 des Grundgesetzes bestimmt den Religionsunterricht als ordentliches Lehrfach. Das deutsche Verfassungsrecht geht von der Religionsfreiheit aus und strebt dessen positive Ermöglichung an. Durch den Religionsunterricht trägt die Schule dazu bei, die Voraussetzungen für die Wahrnehmung dieser Freiheiten zu schaffen ${ }^{4}$.

Klaus Hinkelmann ist Diplom-Soziologe und Theologe. Seit 1975 arbeitet er für die AVMedienzentrale der Erzdiözese München und Freising sowie für die Konferenz der AVMedienzentralen der bayerischen Diözesen. 
Die Arbeit der kirchlichen AV-Medienzentralen legitimiert sich dagegen im Rahmen von Grund- und Ländergesetzen sowie von Konkordaten (z. B. Bayern) doch weitergehend; kaum notwendig zu erwähnen, daß "Religion“ bzw. kirchliche Bildungsarbeit das einzige und ausschließliche Interesse der kirchlichen AV-Medienzentrale sind. Die AV-Medienzentralen als Organe der Kirche spielen den ihnen entsprechenden Teil bei der Sorge der Kirche für den ganzen Menschen; theologisch gesprochen: für sein Heil. Diese Sorge wird konkretisiert in Lehre und Verkündigung einerseits und durch das Angebot einer christlichen Interpretation der Welt an eine pluralistische Gesellschaft andererseits. Dann gilt im Hinblick auf den Einsatz von audio-visuellen Medien: „Wenn Verkündigung immer in der Sprache der Zeit zu geschehen hat und die audio-visuelle Sprache zum Teil die Sprache unserer Zeit ist, müssen wir uns auch in der Verkündigung dieser Sprache bedienen. Von Christus heißt es in der Schrift, daß er in Bildern und Gleichnissen lehrte. Unsere Aufgabe ist es, die Glaubenswahrheiten auch in der Bildsprache unserer Zeit neu auszudrücken." ${ }^{\text {s }}$

Liegt dieser Gedanke gewissermaßen noch auf einem pragmatisch orientierten Niveau, wenn gefordert wird, die Kirche dürfe ihre Aufgaben nicht mit Kommunikationsformen erfüllen wollen, die auf einem technologisch niederem Niveau liegen, als dem allgemeinen Stand der Kommunikationstechniken in der Gesellschaft entspricht, dann bringt die Pastoralinstruktion Communio et Progressio (CP) Ansätze zu einer "Theologie der sozialen Kommunikation und ihrer Instrumente ${ }^{\text {" } 6}$.

Freilich ist die Pastoralinstruktion absichtsvoll allgemein gehalten (CP 3), doch gerade aus dem Grundsätzlichen lassen sich die Wertvorstellungen und Orientierungsvorgaben christlich verantworteten Umgangs mit Medien herausdestillieren. In diesem Grundsätzlichen sind unübersehbar auch die Medien aufgehoben, mit denen die kirchlichen AV-Medienzentralen zu tun haben. Die konkrete Umsetzung der Maxime der Pastoralinstruktion in praktisches Handeln kann $\mathrm{zwar}$ nicht als geleistet gelten, doch der Aufforderungscharakter der Instruktion ist unübersehbar, ist eventuell auch vergleichbar mit dem Impetus des Grundgesetzes.

In diesem weiten Kontext ist Religionsunterricht eine Form kommunikativen Handelns der Kirche, dem allerdings ein hoher Grad an Aufmerksamkeit gewidmet wird. Gerade in den letzten Jahren sind allenthalben breit angelegte Bemühungen zu verzeichnen, die formale, gesetzliche Legitimation des Religionsunterrichts (wieder) in Begründung, Zielsetzung und Inhalt als für die Aufgabenstellung der öffentlichen Schule relevant zu erfüllen.

Die Entwicklung von Curricula und zielorientierten Unterrichtsmitteln hat einen beachtlichen Stand erreicht. Die Aufgabe, audio-visuelle Medien didaktisch verantwortet im Religionsunterricht einzusetzen (statt als Bereicherungsmedien), ist für wohl wenige Fächer so intensiv angegangen worden wie für den Fachbereich Religion. Die Monographien zu diesem Thema nehmen erheblichen Raum ein, im Bereich der Universitäten sind - soweit das von hier aus zu übersehen ist - Bemühungen im Gange, den Theologiestudenten wenigstens spezielle Seminarveranstaltungen anzubieten, die Kirchliche Zentralstelle sowie das Gemeinschaftswerk Evangelischer Publizistik veröffentlichen praxisorientierte Arbeitshilfen, Religionspädagogische Zentren und - im Rahmen ihrer Möglichkeiten - auch die AV-Medienzentralen bieten Lehrerfortbildungen zu Medienfragen an: Das alles sowie die Erfolge der kirchlichen AV-Medienzentralen sprechen eher gegen eine ,im kirchlichen Bereich (festzustellende) Unsicherheit im Einsatz von Medien " ${ }^{\text {. }}$. 


\section{Bereiche einer möglichen Zusammenarbeit zwischen Bildstellen und $A V$-Medienzentralen}

Festzuhalten bleibt zunächst als gesetzlicher Auftrag an die Bildstellen, für Schulen (und Jugend-/Erwachsenenbildung) AV-Medien (AVM) bereitzustellen, oder genauer: die Aufgaben zu erfüllen, die sich aus dem Einsatz von AV-Geräten und AVM für die genannten Bereiche ergeben.

Uberschneidungen mit den Aufgaben kirchlicher AV-Medienzentralen ergeben sich ausschließlich für das Fach Religion; für die Bildstellen ist das nur ein Teilbereich: Sofern man - zur ersten Orientierung - den Film Bild Ton Katalog 77/78 des FWU heranziehen darf, hätten die Bildstellen (bis zu) 33 Fächer abzudecken. Von den nach „Fächern" geordneten 235 Seiten Medienzusammenstellungen dort entfallen 7 Seiten auf „Religion“ mit 156 Titeln.

Die Kirchen haben ein ausgesprochen vitales Interesse am Religionsunterricht, das aus dem eigenen Selbstverständnis heraus lebt, oder besser: aus dem Auftrag ihres Gründers Jesus Christus, wie er in der Geschichte der Theologie erkannt und immer wieder formuliert worden ist. Die kirchlichen AV-Medienzentralen, die dem Auftrag der Kirchen verpflichtet sind, werden in erheblichem Umfang (etwa zu 50-65\%) durch den Religionsunterricht bestimmt. Auch die übrige Arbeit der kirchlichen AVMedienzentralen (kirchliche Bildungsarbeit schlechthin) ordnet sich bruchlos und harmonisch um Gegenstand und Themen des Religionsunterrichts.

So nimmt es denn auch nicht Wunder, daß die Spezialisierung der kirchlichen AVMedienzentralen auf diese Belange der sozialen Kommunikation - soweit sie über audio-visuelle Medien erfolgt, sich in einem entsprechenden Angebot an AVM dokumentiert. Der Deutsche Katechetenverein hat im Zusammenhang und als Vorarbeit ${ }^{8}$ eines Projekts zur EDV-Dokumentation ${ }^{8}$ audio-visueller Medien des FWU eine Zusammenstellung aller im deutschsprachigen Raum für den Religionsunterricht angebotenen AVM erarbeitet; die Zählung ergab rund 2.400 verschiedene Titel. Selbst wenn nach einer ausgesprochen kritischen Sichtung tausend Titel davon ins Archiv verwiesen würden, wird ein außergewöhnlich großer Fundus an Medien erkennbar, der für kirchliche Bildungsarbeit aktiviert wurde ${ }^{10}$.

\section{$Z u$ den Aufwendungen der Bildstellen für das Fach Religion}

Die Aufwendungen aller 487 Bildstellen in der Bundesrepublik für 1976 dürften bei 1 Million DM liegen ${ }^{11}$. Aufschlußreich wäre hier die Aufschlüsselung der Gründe, weshalb die Spanne der Aufwendungen bei den verschiedenen Bildstellen von 0,00 bis $15.000,00$ DM schwankt. Im Durchschnitt ${ }^{12}$ stand 1976 jeder Bildstelle ein Betrag von 2.018,48 DM zur Verfügung: damit können etwa 3-5 Kurzfilme für das Fach Religion angeschafft werden. Die beiden Angaben zusammengenommen lassen erkennen, daß für die Mehrzahl aller Bildstellen die Etats für das Fach Religion ausgesprochen begrenzt sind, daß aber andererseits die tatsächlichen Verhältnisse vor Ort, bei der einzelnen Bildstelle, bedeutend schlechter oder erheblich besser sein können ${ }^{13}$.

Die Rückführung der Gesamtsumme der Ausgaben für das Fach „Religion“ (inclusive der gesetzlich geregelten Jugend- und Erwachsenenbildung) auf den tatsächlichen finanziellen Spielraum der Bildstellen ist in Beziehung zu setzen zu deren Gesamtanschaffungsetats und zu Angaben über die tatsächliche Nutzung der Medien aus dem Fach „Religion“. Die zur Verfügung stehenden Daten ${ }^{14}$ lassen bedauerlicherweise 
exakte Berechnungen nicht zu, konvergieren jedoch alle um eine Wertspanne. Der Schluß ist - mit gebotener Zurückhaltung - erlaubt, davon auszugehen, daß im Mittel der "Religions“-Etat 5-7\% des Gesamtetats einer Bildstelle betragen dürfte. Unterstellt man, daß das finanzielle Engagement mit der tatsächlichen Nutzung (Ausleihe) der Religions-Medien korrespondiert, ist die durchschnittliche Ausleihhäufigkeit pro Bildstelle mit \pm 800 Ausleihen $^{15}$ für 1976 anzusetzen.

Alles in allem wird begründet vermutet werden dürfen, daß die Bildstellen innerhalb ihrer Aufgaben den Proporz wahren. Dies kann aber auch so gesehen werden, daß "Religion" für die Bildstellen aller Wahrscheinlichkeit nach nur die Bedeutung hat, die sich an Aufwand und Verleih ablesen läßt.

\section{Zum finanziellen Engagement der Kirchen bei den Bildstellen und beim FWU}

Die - gemessen am Bedarf - offenkundig nicht sehr weiten Etat-Grenzen der Bildstellen legen bei den Interessierten die Frage nahe, ob nicht die Kirchen lieber Geld an die Bildstellen geben sollten ${ }^{16}$, statt eigene Verleihorganisationen aufzubauen. Faktisch verfahren die Kirchen zweigleisig: In Einzelfällen gibt es Zuwendungen an einzelne Bildstellen ${ }^{17}$, ein Produktionszuschuß von $100.000 \mathrm{DM}$ geht an das FWU ${ }^{17}$. Hinzuzurechnen sind auch die Gehälter der beiden kirchlichen Referenten für Religion beim (!) FWU (Herr May für evangelische, Herr Täubl für katholische Religion), alles in allem ein Betrag von rd. $200.000 \mathrm{DM}^{18}$. Das entspricht vielleicht dem Sach-Etat einer gut ausgebauten kirchlichen AV-Medienzentrale.

Tatsächlich hatten und haben die Kirchen gute Gründe, selber eine Verleihorganisation für die kirchliche Bildungsarbeit zu forcieren. Die kirchlichen AV-Medienzentralen verstehen sich keineswegs als "Materialstellen“; die Verwaltung des Materials bezeichnet nur einen Teil der Aufgaben. Wesentlich für kirchliche AV-Medienzentralen ist der Auf- und Ausbau einer Fachberatung, die den differenzierten Angeboten kirchlicher Bildungseinrichtungen und dem diffizilen Charakter von "Religion" von der Medienseite her gerecht wird. Soweit die Medien nicht Beiträge zu offenkundig religiösen Themen des Religionsunterrichts leisten (z. B. Bibelkunde, Gottesfrage usw.) beziehen sie ihre Rechtfertigung für den Einsatz in Religionsunterricht und kirchlicher Bildungsarbeit nur selten aus religiösen Handlungen, Symbolen u. ä. Vielmehr bieten sie - oft im Vorfeld jeglicher theologischer Thematik - Fragestellungen, Lebenserfahrungen und Reflexionen, die erst in die religiöse und theologische Fragestellung erhoben werden müssen.

Auch ist der didaktische Stellenwert dieser AVM nicht so eindeutig wie vielleicht in anderen Bereichen. Er ist zu bestimmen aus der Konzeption des Mediums und aus den daran geknüpften Aufgaben. Die Beratung hat ebenfalls zu berücksichtigen, daß die Mehrzahl der Medien - besonders der Kurzfilm - letztlich unter bestimmten Interessen produziert wurden, die oft wenig mit dem Einsatz in Schule oder Bildungsarbeit gemein haben. Nur selten sind sie auf bestimmte Lehrpläne hin konzipiert. Die Probleme, die sich daraus für einen didaktisch sinnvollen Einsatz ergeben, können von den AV-Medienzentralen zwar auch letztlich nicht gelöst werden, doch kann eine qualifizierte Beratung z. B. falschen Erwartungen die Spitze nehmen und Entscheidungshilfe sein. Dieser Erkenntnis dient die Einrichtung eines Zertifikatskurses der kirchlichen Zentralstelle für Medien für Mitarbeiter in AV-Medienzentralen, die auf die Vermittlung fachlicher Kompetenz gerade in diesem Bereich besonderen Wert legt. 
Vorstellungen, die auf eine Angliederung oder Integration der kirchlichen AVMedienzentralen an das Bildstellenwesen zielen ${ }^{10}$, lassen eine Reflexion über derartige strukturelle Unterschiede vermissen und geben auch keine Antwort über die Konsequenzen solcher Maßnahmen: Unvermeidlich wären höchstwahrscheinlich Anpassungen an die Organisation des Bildstellenwesens. Könnten die kirchlichen AV-Medienzentralen unter einem solchen Dach dem ihnen gemäßen Selbstverständnis gerecht werden, den als richtig erkannten Weg im Verleih und beim Service weiter ausbauen? Oder wäre das nicht ein Weg, aus AV-Medienzentralen tatsächlich "Materialstellen“ zu machen?

\section{Das Verhältnis von Bildstellenwesen zu kirchlichen $A V$-Medienzentralen als Begegnung zwischen Staat und Kirche}

Die angedeuteten Uberlegungen pragmatisch-struktureller Natur haben ihr eigenes Gewicht, werden aber überlagert und gestützt durch grundsätzlichere Erwägungen.

In dem Verhältnis Bildstellen-AV-Medienzentralen findet sich ein Stückchen der Beziehung Kirche-Staat. Demzufolge können die Regeln, die in diesem Lande für den wohl immer etwas gespannten Umgang der beiden Bereiche ausgebildet wurden, entsprechende Anwendung finden. Die im Grundgesetz verankerten Bestimmungen laufen auf eine Formel hinaus, die bei grundsätzlicher Trennung von Kirche und Staat sachorientierte Zusammenarbeit nicht ausschließt. Der moderne Staat ist Sozialstaat. Von seinen umfassenden staatlichen Verpflichtungen her kommt ihm eine entsprechend umfassende Kompetenz zu. Trotz dieser Generalkompetenz muß der Staat jedoch nicht selber "gleichzeitig und überall zugunsten des Wohls des einzelnen und der Gemeinschaft eingreifen “20. Bevor er selber eingreift, hat er die Anstrengungen der gesellschaftlichen Gruppen „anzuerkennen und zu fördern“"20. Einzugreifen hätte - bei seiner unbestrittenen Kompetenz der Staat dort, wo die gesellschaftlichen Kräfte, die den Staat letztlich aufbauen, die Aufgaben für das Gemeinwohl nicht wahrnehmen können - das alt-bewährte Subsidiaritätsprinzip. Und gerade in der Medienarbeit, bei der Distribution audio-visueller Medien für die Bildungsbereiche, zeigen sich sehr schnell die Grenzen des staatlichen/kommunalen Apparates, seine Generalkompetenz im Sinne einer weitgehenden Bedarfsdeckung zu füllen ${ }^{21}$. Der Schritt, anzuerkennen, daß auch die kirchlichen AV-Medienzentralen - wie andere freie Träger - bedeutsame Arbeit in der Gesellschaft leisten, kann dann nicht mehr so schwer sein.

Im Sinne des Subsidiaritätsprinzips könnte fundiert die Meinung vertreten werden, daß umgekehrt - statt finanzieller Zuwendungen der Kirchen an die staatlichen Organe oder statt der Einbeziehung der kirchlichen AV-Medienzentralen in die Bildstellen - der Staat und seine Organe die freien Träger unterstützen könnten. Andererseits braucht wohl kaum betont zu wenden, daß die Kirche ihrerseits nicht das Recht hat, den Staat (und seine Organe) aus seiner (ihrer) Pflicht zu entlassen.

Es darf wohl auch gefragt werden, ob nicht ein plurales System verschiedener Verleihorganisationen dem Gemeinwesen mehr von Nutzen ist als ein einheitlich konzipiertes. Das differenzierte Angebot an Medien und an Leistung mit unterschiedlichen Schwerpunkten, wie es derzeit durch Bildstellen, Landesfilmdienste, kirchliche AVMedienzentralen und einige spezielle Verleihorganisationen, wie z.B. Deutsches Film-Zentrum u.a., gekennzeichnet ist, ermöglicht eine differenzierte Orientierung am Entleiher, als bei nur einem System zu erzielen wäre. 
Als Hauptakzente, die je nach örtlicher Gegebenheit durchaus Verschiebungen und Nebenakzente zulassen, könnte man die Schwerpunkte der Arbeit vielleicht grob unterteilen:

Bildstellen

Landesfilmdienste

AV-Medienzentralen
Schulwesen

außerschulischer Bereich

schulischer Religionsunterricht und kirchliche Bildungsarbeit überhaupt

(daneben: Verleihstellen für bestimmte andere Aufgaben wie z. B. Landes- und Bundeszentralen für politische Bildung / Deutsches Filmzentrum u. a.)

Überschneidungen sollten ausdrücklich sogar erwünscht sein. Konkurrenz auf dem je eigenen Gebiet wird vermutlich "Tatkraft und Phantasie“22 aller Beteiligten zugunsten der Entleiher beflügeln. Wohl kaum eine Maßnahme wäre so unangebracht wie das exklusive Vereinnahmen von Kompetenzen.

\section{Zusammenfassung}

Kirchliche AV-Medienzentralen verstehen sich als ein spezialisierter Service für die Belange der kirchlichen Bildungsarbeit. Die traditionell weitgespannten Themen dieses Bereiches führen zu Berührungspunkten mit anderen Bildungseinrichtungen und Distributionsstellen für alle Bereiche der Medienarbeit. Eine Zusammenarbeit in den Bereichen gemeinsamen Interesses sollte angestrebt werden, erscheint jedoch nur möglich unter Anerkennung der gegenseitigen Grenzen.

Austausch von Informationen und gemeinsame Gespräche auf der Ebene BildstellenAV-Medienzentralen sollten ohne Probleme möglich sein. Hier können auch die örtlichen Gegebenheiten präzise bestimmt wenden.

Eine sehr konkrete Möglichkeit der Zusammenarbeit ist z. B. auf der Ebene der Landesbildstellen gegeben. In der Generalstudie bei Schorb heißt es:

„Zulassung von Medien im Unterricht. Bestimmte audio-visuelle Medien dürfen ohne Zulassung nicht im Unterricht eingesetzt werden. Dies gilt insbesondere für Unterrichtsfilme. Alle im Katalog des Instituts für Film und Bild aufgeführten Filme haben die Zulassung. Von den Schulen sind grundsätzlich diese Filme zu verwenden. Andere Filme (z. B. Industriefilme, Werbefilme, Filme des Landesfilmdienstes usw.) können eingesetzt werden, wenn sie von der Landesbildstelle zugelassen worden sind. Für noch nicht zugelassene Unterrichtsfilme kann ein entsprechender Antrag an die Landesbildstelle gerichtet werden. ${ }^{\text {"23 }}$ Diese Bestimmungen gelten jedenfalls für den Freistaat Bayern. Für das Fach „Religion“ wären hier die Landesbildstellen aufgerufen, das Fachwissen der kirchlichen AV-Medienzentralen gezielt anzufordern und diese Aufgabe gemeinsam anzugehen. Die Zusammenarbeit sollte in dieser Sicht beim Konkreten im Sinne einer „Politik der kleinen Schritte“ beginnen.

Festzuhalten bleibt ebenfalls, daß sich niemand um „Selbstverständnis und Aufgabenbeschreibung der kirchlichen Einrichtungen im AV-Bereich“24 größere Sorgen zu machen braucht. Vielleicht ist die Frage nur die, daß kirchliche Stellen ihr „Selbstverständnis" nicht zureichend in der Offentlichkeit vertreten, weil sie mit der alltäglichen Kleinarbeit eingedeckt sind und darüber hinaus die politische Dimension vernachlässigen. 
1. Vg1.: „Die Situation der AV-Medienarbeit der Katholischen Kirche in der Bundesrepublik Deutschland und Berlin (West) " - Bericht - Hrsg. Zentralstelle Medien der Deutschen Bischofskonferenz, Bonn 1977, S. $30 \mathrm{ff}$.; Anton Täubl erläuterte in seinem Beitrag in "Communicatio Socialis" 3/77, S. 209-219, eine Umfrage bei den Bildstellen zu dem Verhältnis (Landes-, Kreis-, Stadt-) Bildstellen - evangelische und katholische AV-Medienzentralen; der Artikel stellt der Offentlichkeit über die Erläuterung hinaus Interpretationen der Daten und vor allem die Sicht Täubls über die Konsequenzen seiner Interpretation für die Beziehung $\mathrm{zw}$ wschen den beiden Medienverleihorganisationen vor.

So klar Täubl die Forderungen und Erwartungen der FWU und der Bildstellen als zwingend herausstellt: die Gemeinsamkeiten und die Unterschiede der beiden Verleihorganisationen erarbeitet er ebenso wenig, wie er die Bedingungen für die Möglichkeit einer Kooperation adäquat erfaßt.

2. Vgl. Täubl: a.a.O. 209 / FWU-Katalog S. 2 u. $4 /$ ebenso Schorb: Generalstudie zur Ermittlung und Deckung des Bedarfs an audiovisuellen Medien (Zwischenergebnisse) Rechtsfragen; Herausgeber: Staatsinstitut für Bildungsforschung und Bildungsplanung, München 1975, Anlage 2 und öfter.

3. Vgl. Täubl: a.a.O. 209.

4. Vgl. auch: Der Religionsunterricht in der Schule. Beschluß der gemeinsamen Synode der Bistümer in der Bundesrepublik Deutschland, Synodenbeschlüsse 4.

5. Julius Kardinal Döpfner: Vorwort zum Katalog „film bild ton “ der Konferenz der AV-Medienzentralen der bayerischen Diözesen.

6. Vgl. dazu H. Wagner: Einführung und Kommentar (zu CP), in: Päpstliche Kommission für die Instrumente der sozialen Kommunikation, Pastoralinstruktion Communio et Progressio, Trier 1971, S. 18 fff.; vgl. Bericht Zentralstelle Medien, a.a.O., S. 22-27.

7. Täubl: a.a.O. S. 214. Mit den Ergebnissen der Umfrage - soweit sie dort zu ersehen sind - läßt sich eine solche Behauptung nicht belegen.

8. Das FWU war davon ausgegangen, die vermeintlich geringen Bestände an AVM für den Religionsunterricht komplett dokumentieren zu können. Nach dieser Vorarbeit wurden zunächst (und bis auf weiteres) nur die FWU-Medien für Religion dokumentiert.

9. Dr. R. Marczinski auf der Visodata, München Nov. 1975.

10. Um Mißverständnissen und Fehlinterpretationen vorzubeugen: es soll und kann hier keinesfalls darum gehen, die Leistungen der ${ }_{n}$ größte(n) Verleihorganisation für AVMedien im nichtkommerziellen Bereich" (Täubl: a.a.O. S. 209) herabzusetzen. Hier soll - soweit die Daten das zulassen - nachgeholt werden, die Bereiche halbwegs präzise auszuloten und den Umfang dessen auszumachen, worüber Bildstellen und kirchliche Verleihorganisationen miteinander reden können.

11. Die Berechnung erfolgte anhand der Angaben Täubls, a.a.O. S. 211 u. a.: $663.314,03 \mathrm{DM}$ für Religion aus 327 eingegangenen Fragebögen entspricht 2.018,48 DM durchschnittlich. Dieser Durchschnitt multipliziert mit 487 Bildstellen ergibt 991.926,72 DM (Extrapolation des Mittelwertes).

12. Gemeint ist das arithmetische Mittel; zur Berechnung siehe Anmerkung 11. Zur Eigenart dieses Mittels gehört es, daß extreme Werte stark in den Mittelwert eingehen.

13. Auch von Region zu Region streuen die Mittel erheblich.

Vgl. Täubl: a.a.O. S. 211: LBS-Bereich Württemberg 130.867,48 DM bei 27 Bildstellen $(=4.846,94 \mathrm{DM} \phi)$; LBS-Bereich Nordbayern $36.832,05 \mathrm{DM}$ auf 29 Bildstellen $(=1.270,07 \mathrm{DM} \phi)$.

14. Nicht im Sinne einer exakten Beweisführung, sondern nach Art eines „Konvergenzbeweises" sollen folgende Überlegungen dienen:

a) "Religion" ist eines von dreiunddreißig Fächern aus den Sachgebieten des FWUKatalogs. Sieben von 235 Seiten betreffen das Fach Religion, das macht rund $3 \%$ des Gesamtangebots aus. (Wobei nicht unterstellt ist, daß sich das FWU-Angebot auf alle Fächer gleichmäßig zu verteilen habe. Ebenso ist klar, daß das FWU-Angebot für die Bildstellen nicht verbindlich ist.)

b) Der Kopienverkauf des FWU 1976 hatte ein Volumen von 19 Millionen DM (davon 
14 Millionen für schulische Zwecke) (Quelle: Täubl: a.a.O. S. 211). Bezieht man die geschätzten Gesamtausgaben aller Bildstellen (vgl. Anm. 11) auf diese Zahlen - obwohl die Bildstellen auch andere Einkaufsquellen nutzen - errechnet sich ein Anteil von $5,22 \%$ (bzw. 7,08\%) für das Fach „Religion“.

c) Eine andere Umfrage des FWU bei 58 Kreis- und Stadtbildstellen, die wiederum "Bildstellen aus einem mittleren Bereich repräsentieren", beziffert die durchschnittlichen Beschaffungsmittel je Bildstelle auf 55.330 DM (Spanne: 5.000-18.000 DM). Nimmt man diese Zahl als Maßstab, so macht das arithmetische Mittel aus Täubls Umfrage 3,67\% der durchschnittlichen Beschaffungsmittel für das Fach „Religion“ aus.

Quelle: Das Interview, in: AV-Informationen 77/2 S. 4/5 Hrsg. FWU. Der Vergleich $\mathrm{zweier}$ Umfragen ist allerdings problematisch.

15. Das Interview a.a.O.

16. So Täubl: a.a.O. S. 213 ( $2 \mathrm{mal}) / 215$.

17. Ebd. S. 213; vgl. auch Bericht Zentralstelle Medien, a.a.O., S. 19.

18. Als ausgesprochen unglücklich zu werten ist in diesem Zusammenhang eine Aufrechnung der Anschaffungen der Bildstellen für Religion gegenüber den o. g. Leistungen der Kirchen (Täubl S. 213). Täubl schreibt: „Die finanziellen Zuwendungen der Kirchen ... nehmen sich gegenüber den Ausgaben der Bildstellen für kircbliche Zwecke (Hervorhebung durch den Verfasser) ... nur bescheiden aus." Hier erweckt er den Eindruck, die beiden Kirchen seien "Trittbrettfahrer" der staatlichen bzw. kommunalen Einrichtungen der Bildstellen. Hieran knüpft er vorsichtig die Forderung nach "finanziellen Zuwendungen seitens der Kirchen" unter dem Mantel der "Kooperation“. Ein gewisses Mißverständnis kommt hier dadurch zustande, daß plötzlich nicht mehr vom ordentlichen Lehrfach Religion oder von den gesetzlichen Bestimmungen der Jugend- oder Erwachsenenbildung die Rede ist, sondern von "kirchlichen Zwecken ${ }^{*}$ gesprochen wird.

19. So Täubl: a.a.O. S. 213.

20. Aufgaben der Kirche in Staat und Gesellschaft, Arbeitspapier der Sachkommission V der Gemeinsamen Synode der Bistümer in der Bundesrepublik Deutschland, Sonderdruck aus „Synode" $1 / 73$, S. 18.

21. Die Ansprüche des Staates, seine Kompetenzen zuungunsten der freien Träger in Anspruch zu nehmen, haben in anderen Fällen (z. B. bei den Kindergärten oder den Krankenhäusern) nicht unbedingt das Gemeinwohl gemehrt. Die Vorstellung einer Integration angesichts der Bestrebungen, kirchliche und staatliche Belange nicht zu vermengen bzw. sogar die Trennung beider Bereiche noch deutlicher zu vollziehen, liegt deutlich außerhalb des generellen politischen Trends. Nun gibt es durchaus erfolgreiche Versuche, bei denen Verleihorganisationen unter einem Dach zusammenarbeiten: beim Landesfilmdienst Mainz ist die AV-Medienzentrale Untermieter, in Würzburg ist es umgekehrt. Es ist jedoch gewiß kein Zufall, daß sich hier zwei freie Träger zusammengeschlossen haben. In Nürnberg haben sich allerdings die Evangelische Medienzentrale und der Landesfilmdienst wieder getrennt - u. a. auch wohl deshalb, weil sich herausstellte, daß die Entwicklung beider Einrichtungen auf die Dauer unterschiedliche Wege nahm.

22. Täubl: a.a.O. S. 216.

23. Schorb: a.a.O. 8.9.

24. Täubl: a.a.O. S. 216.

\section{S U M M A R Y}

In addition to the State film and media centres, AV Media Centres of the Church have, in recent years, developed most positively. The question has now to be raised as to how they fit into the overall framework of these activities. Along with State film and media centres, the Church centres are responsible for AV material for religious instruction in state 
schools. Apart from the tasks which they have in common there are also some differences in structure, aim, and legal aspects, which should not be overlooked, otherwise this could lead to a premature demand for amalgamation of Church centres with those of the State, which do not answer the various explicit needs of the Church. The Church centres have to help her realise her values, her interpretation of the world, and her proclamation of the good news to modern man. The author pleads for separate media and services through the distribution organisations, according to needs of local users. Co-operation between the distributors and users should be organised according to the needs and opportunities 'sur place'. This should be possible if they are guided by concrete local needs of the project.

\section{RESUMÉ}

Les centrales des moyens audio-visuels de l'Eglise ont montré un développement constant et bien couronné de succès à côté de leurs grands frères - les services nationaux de prêt de photos et de films. La question concernant leur valeur en tant qu'autorité et leur place dans les systèmes de prêt non-commercial pour les moyens audio-visuels se conçoit aisément. Avec les services de prêt de photos, les centrales de moyens audio-visuels se partagent surtout la tâche d'approvionner en moyens audio-visuels l'enseignement religieux dans les écoles publiques. Cependant, malgré les communautés données, on ne peut négliger les différences d'organisation, de but et de légitimation des services de prêt; sinon persiste le danger qu'on ne réclame une standardisation qui ne serait pas conforme à une demande différenciée. L'article souligne le fait que les centrales des moyens audio-visuels de l'Eglise ont de toute façon une part conforme à la tâche de l'Eglise: apporter à l'homme d'aujourd'hui la représentation chrétienne des valeurs, l'interprétation du monde et la proclamation. L'article plaide pour une offre différenciée en moyens et en services par l'intermédiaire des organisations de prêt - correspondant aux différentes situations de demandes des utilisateurs. La coopération entre les organisations et les représentants devrait être possible selon les besoins du lieu de façon concrête et "orientée vers le projet", sans aucune difficulté.

\section{RESUMEN}

Las centrales eclesiales de medios audiovisuales (AV) experimentaron durante los últimos años, junto a sus hermanos mayores, los servicios fotográficos y fílmicos, un desarrollo permanente y exitoso. Ello plantea la cuestión de su valor y ubicación entre las distribuidoras no-comerciales de medios audiovisuales. Junto con los servicios fotográficos, las centrales AV comparten sobre todo la misión de suministrar material audiovisual a las clases de religión en las escuelas públicas. Pero, a pesar de las coincidencias, no pueden olvidarse las diferencias existentes en la estructura, meta y legitimación de las distribuidoras; de lo contrario se corre el peligro de una precipitada aspiración a la fusión, que no correspondería a la demanda diferenciada. El artículo destaca que las centrales AV eclesiales cumplen una parte proporcional de la misión de la Iglesia: presentación de valores cristianos, interpretación del mundo y proclamación del Evangelio al hombre de hoy. El artículo propone una oferta diferenciada de medios y servicios mediante organizaciones de distribución de acuerdo con la diferente demanda. La cooperación entre organizaciones y agentes debería ser posible sin grandes dificultades segun las necesidades locales concretas y "proyectadas «. 